\title{
Two Color Matter in the Quenched Approximation
}

\author{
Pietro Giudice* \\ Dipartimento di Fisica Teorica, Università di Torino and INFN, sezione di Torino, Italy \\ E-mail: giudicedto.infn.it
}

\section{Simon Hands}

Physics Department, Swansea University, Singleton Park, Swansea SA2 8PP, United Kingdom

E-mail: S.hands@swan.ac.uk

We study a quenched SU(2) lattice gauge theory in $4 \mathrm{~d}$ in which the spatial gauge ensemble $\left\{U_{i}\right\}$ is generated from a $3 \mathrm{~d}$ gauge-Higgs model and the timelike link variables are "reconstructed" from the Higgs fields. The resulting ensemble is used to study quenched quark propagation with non-zero chemical potential $\mu$. While it proves possible to alter the strength of the inter-quark interaction by changing the parameters of the dimensionally reduced model, there is no evidence for any region of parameter space where quarks exhibit deconfined behaviour or thermodynamic observables scale as if there were a Fermi surface.

The XXV International Symposium on Lattice Field Theory

July 30 - August 42007

Regensburg, Germany

\footnotetext{
* Speaker.
} 


\section{Introduction}

Lattice QCD suffers from the so-called Sign Problem; the Lagrangian density with $N_{f}$ quark flavors has the form $\bar{q} M q$; the functional measure $\operatorname{det}^{N_{f}} M(\mu)=\operatorname{det}^{N_{f}} M^{*}(-\mu)$ implies that for $\mu \neq 0$ the action is complex, rendering Monte Carlo importance sampling impracticable.

Can one at least perform lattice QCD simulations in the quenched $N_{f} \rightarrow 0$ limit, i.e. study the propagation of valence quarks with $\mu \neq 0$ through a non-perturbative gluon background [1]?

Of course, the information extracted from such an approach could be at best qualitative, since (unlike the case of $T>0$ ) the gauge field ensemble $\left\{U_{\mu}\right\}$ can only respond to $\mu \neq 0$ via virtual quark loops. Anyway, such information might be valuable in furnishing a non-perturbative definition of the Fermi surface, whose existence is assumed in most phenomenological treatments of dense matter.

In the context of a random matrix theory, Stephanov [2] showed that the quenched theory should be thought of as the $N_{f} \rightarrow 0$ limit of a QCD-like theory with not just $N_{f}$ flavors of quark $q \in \mathbf{3}$ of the SU(3) gauge group but also with $N_{f}$ flavors of conjugate quark $q^{c} \in \overline{\mathbf{3}}$. As a consequence, $q q^{c}$ bound states appear in the spectrum, resulting in baryons degenerate with light mesons. For $\mu / T \gg 1$ there is an onset transition from the vacuum to a ground state with quark number density $n_{q}>0$. For QCD it occurs at $\mu_{o} \simeq m_{N} / 3$ ( $m_{N}$ is the nucleon mass) but if conjugate quarks are present $\mu_{o} \simeq m_{\pi} / 2$.

We try to modify the gluon background in some way so that color confinement no longer holds: then the role of $q q^{c}$ excitations may not be so important in determining the ground state in the quark sector. Our hope is that valence quark propagation in such a background may qualitatively resemble that of the deconfined regime of the phase diagram at $\mu / T \gg 1$. We start with the $3 d$ configurations characteristic of the deconfined phase found at $T>T_{c}, \mu / T \ll 1$ produced by the approach to hot gauge theory known as Dimensional Reduction (DR). The quenched action is the $3 d \mathrm{SU}(2)$ gauge - adjoint Higgs model obtained by DR from $4 d$ SU(2) given by Eqn. (4) of Ref.[3]:

$$
S_{3 d}=\beta \sum_{x, i>j}\left(1-\frac{1}{2} \operatorname{tr} U_{x, i j}\right)+2 \sum_{x} \operatorname{tr}\left(\varphi_{x} \varphi_{x}\right)-2 \kappa \sum_{x, i} \operatorname{tr}\left(\varphi_{x} U_{x, i} \varphi_{x+\hat{\imath}} U_{x, i}^{\dagger}\right)+\lambda \sum_{x}\left(2 \operatorname{tr}\left(\varphi_{x} \varphi_{x}\right)-1\right)^{2},
$$

where $\varphi \equiv \frac{1}{2} \varphi_{a} \tau_{a}$ represents the adjoint Higgs field. In the DR approach, all non-static modes of the gauge theory are integrated out leaving a $3 d$ gauge-Higgs model describing the non-perturbative behaviour of the remaining static modes.

Our goal is to study quark propagation through a non-confining quenched gluon background with $\mu \neq 0$. Since chemical potential couples to quarks via the timelike component of the current $\mu \bar{\psi} \gamma_{0} \psi$, this is an inherently four-dimensional problem. In order to generate such a background we take a $3 d$ configuration generated by the DR simulation, motivated by the fact that it describes deconfining physics, and "reconstruct" the gauge field in the timelike direction via the prescription:

$$
U_{0}=\exp \left(i g \sqrt{\frac{\kappa}{N_{\tau}}} \varphi\right)=\cos \left(\tilde{g} \sqrt{\varphi_{a} \varphi_{a}}\right)+i \frac{\tau_{a} \varphi_{a}}{\sqrt{\varphi_{a} \varphi_{a}}} \sin \left(\tilde{g} \sqrt{\varphi_{a} \varphi_{a}}\right),
$$

with $\tilde{g}=\sqrt{\frac{\kappa}{\beta}}$. Spatial link variables $U_{i}$ are taken to be time independent and identical to their $3 d$ counterparts. Henceforth we use $4 d$ configurations $\left\{U_{\mu}\right\}$ generated as outlined above as input in quenched studies. 
Since $q$ and $\bar{q}$ fall in equivalent representations of the gauge group hadron multiplets contain both $q \bar{q}$ mesons and $q q, \bar{q} \bar{q}$ baryons, which are degenerate at $\mu=0$. In the chiral limit the lightest hadrons are Goldstone bosons and can be analysed using chiral perturbation theory $(\chi \mathrm{PT})$ [㺻: at leading order for $\mu \gg T$ a second order onset transition from vacuum to matter consisting of tightly bound diquark scalar bosons is predicted at exactly $\mu_{o}=m_{\pi} / 2$. In the limit $\mu \rightarrow \mu_{o+}$ the matter in the ground state becomes arbitrarily dilute, weakly-interacting and non-relativistic: a textbook example of Bose-Einstein condensation. At the same point the chiral condensate $\langle\bar{q} q\rangle$ starts to fall below its vacuum value and a non-vanishing diquark condensate $\langle q q\rangle$ develops. The diquark condensate spontaneously breaks U(1) baryon number symmetry, so the ground state is superfluid.

More recent simulations have found evidence for a second transition at larger $\mu$ to a deconfined phase, as evidenced by a non-vanishing Polyakov loop [5] and by a fall in the topological susceptibility [6]. In this regime thermodynamic quantities scale according to the expectations of free field theory (also referred to as "Stefan-Boltzmann" (SB) scaling), namely $n_{q} \propto \mu^{3}$, and energy density $\varepsilon \propto \mu^{4}[5]$.

\section{Numerical Results}

We have chosen $\beta=9.0$ sufficiently close to the continuum limit for the DR formalism to be trustworthy, and start with a point with $T=2 T_{c}$ : this corresponds to $\kappa=0.3620027, \lambda=$ 0.0020531. It is important to make a precise determination of the pion mass at $\mu=0$ : we obtain $m_{\pi} a_{t}=0.2321(1)$ on a $8^{3} \times 32$. Since this scale is not too dissimilar to $L_{s}^{-1}$, we have repeated the measurement on $16^{3} \times 64$, where we find $m_{\pi} a_{t}=0.2368(3)$. The systematic error due to finite volume is significant, but small enough at $2 \%$ to be acceptable for this exploratory study. We have analysed the quark density $n_{q}$ and chiral condensate $\langle\bar{q} q\rangle$ as functions of $\mu$ for various $j$ with $0 \leq \mu / T \leq 8$. There is a transition at $\mu a_{t} \approx 0.12$, becoming more abrupt as diquark source $j \rightarrow 0$, from a phase with $n_{q}=0,\langle\bar{q} q\rangle$ constant to one in which $n_{q}$ increases approximately linearly with $\mu$ and $\langle\bar{q} q\rangle \propto \mu^{-2}$. This is in complete accordance with the scenario described by $\chi$ PT in which as $\mu$ increases at $T \approx 0$ there is a transition at $\mu_{c}=m_{\pi} / 2$ from the vacuum to a weakly-interacting Bose gas formed from scalar diquarks. The diquarks are supposed to Bose-condense to form a superfluid condensate; on a finite system this must be checked at $j \neq 0$ using the $\left\langle q q_{+}\right\rangle$observable: the condensate increasing monotonically with $\mu$. To determine the nature of the ground state an extrapolation $j \rightarrow 0$ is needed. We have used a cubic polynomial for data with $0.02 \leq j a \leq 0.1$; Fig. 11 confirms that once again there is an abrupt change of behaviour in the order parameter at $\mu \approx$ $m_{\pi} / 2$, and that the high $-\mu$ phase is superfluid. Next we explored a parameter set corresponding to a smaller scalar "stiffness" by changing to $\kappa=0.1$. Naively this corresponds to a huge value of $T / T_{c}$, ie. taking us further into the deconfined phase of the hot gauge theory. Of course, whether DRbased concepts remain valid for the reconstructed theory must be addressed empirically. This time we used a volume $8^{3} \times 64$ for the bulk observables and at $\mu=0$ determined the pion mass $m_{\pi} a_{t}=$ $0.1377(1)$ on $8^{3} \times 128$, and $m_{\pi} a_{t}=0.1423(4)$ on $16^{3} \times 64$, showing that the finite volume error is now roughly $3 \%$. We studied $n_{q}$ and $\langle\bar{q} q\rangle$ as functions of $\mu$ for $0 \leq \mu / T \approx 12$. It is noteworthy that for $\mu>m_{\pi} / 2 n_{q}(\mu)$ is numerically very similar to the values found at $\kappa=0.3620027$, whereas for $\mu<m_{\pi} / 2$ the chiral condensate $\langle\bar{q} q\rangle$ is significantly smaller, indicative of a weaker quark anti-quark binding at this smaller $\kappa$. Moreover, using the Gell-Mann-Oakes-Renner relation, valid 


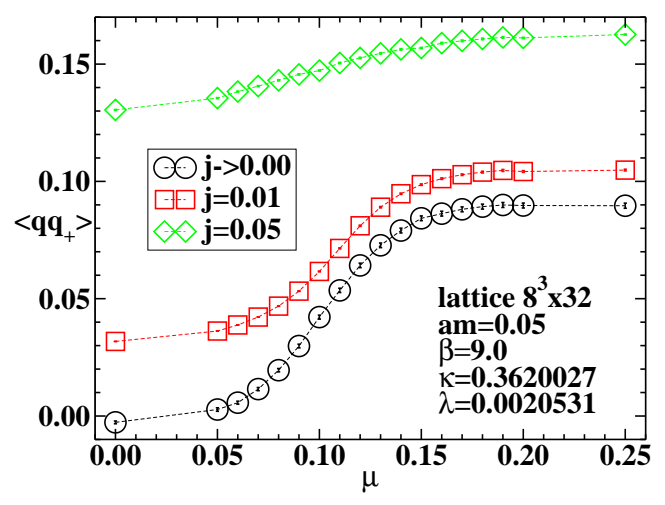

Figure 1: $\left\langle q q_{+}\right\rangle$vs. $\mu a_{t}$ for various $j$; the extrapolation to $j \rightarrow 0$ is obtained using a cubic polynomial.

for $\chi \mathrm{PT}, f_{\pi}^{2} m_{\pi}^{2}=2 m_{q}\langle\bar{q} q\rangle$ we have: $f_{\pi}^{2} a^{2}=0.99$ for $\kappa=0.1$ and $f_{\pi}^{2} a^{2}=0.58$ for $\kappa=0.3620027$. The non-interacting limit is $f_{\pi} \rightarrow \infty$ therefore the interparticle intractions are weaker at $\kappa=0.1$. As before, there is a clear discontinuity in the observables' behaviour at $\mu_{c} \simeq m_{\pi} / 2$, and the general picture is qualitatively very similar, suggesting that the $\chi \mathrm{PT}$ scenario is still applicable. Diquark binding is now also much weaker.

It is disappointing that we have found no qualitative change in physics as the parameters are varied - recall that the $\chi \mathrm{PT}$ model which describes the results reasonably well is based on the assumption of confinement, or at least on the presence of very tightly bound diquark states in the spectrum.

To explore the parameter space more widely we focussed on a single observable, $n_{q}$, and scanned the $(\kappa, \lambda)$ plane on $8^{3} \times 16$ at five different values of $\mu$ with $\beta=9.0, m a=0.05$ and $j a=0.01$. Data shows that except for $\lambda=0.1$ the results for fixed $\mu$ are practically independent of $\kappa$ and of $\lambda$. Moreover, $n_{q}$ increases linearly with $\mu$ over a wide region of parameter space, as it does for $\mu>\mu_{c}$. This approximate linear behaviour is once again a prediction of $\chi \mathrm{PT}$, and is to be contrasted with the $n_{q} \propto \mu^{3}$ behaviour expected of a deconfined theory where baryons can be identified with degenerate quark states occupying a Fermi sphere of radius $k_{F} \approx \mu$. The absence of this scaling is a further reason to conclude that the reconstructed model does not describe deconfined physics.

\subsection{Bosonic Spectrum}

An appropriate set of states to look at, to study the bosonic spectrum, is constituted of pion, scalar, higgs and goldstone. Pion and scalar states are related via the $\mathrm{U}(1)_{\varepsilon}$ global symmetry $\chi \mapsto$ $e^{i \alpha \varepsilon} \chi, \bar{\chi} \mapsto \bar{\chi} e^{i \alpha \varepsilon}$. Analogous to chiral symmetry for continuum spinors, this is an exact symmetry of the action in the limit $m \rightarrow 0$. In a phase with spontaneously broken chiral symmetry, the scalar is massive, and the pion a Goldstone mode, becoming massless as $m \rightarrow 0$. Similarly, "higgs" and "goldstone" diquark states are related via the $\mathrm{U}(1)_{B}$ baryon number rotation $\chi \mapsto e^{i \beta} \chi, \bar{\chi} \mapsto \bar{\chi} e^{-i \beta}$, an exact symmetry of the action in the limit $j \rightarrow 0$. In a superfluid phase with $\left\langle q q_{+}\right\rangle \neq 0$, the higgs is massive, and the goldstone massless in the limit $j \rightarrow 0$.

The boson correlators are constructed from the Gor'kov propagator where appear the $2 \times 2$ (in color space) matrices $N \sim\left\langle\chi_{x} \bar{\chi}_{y}\right\rangle$ and $A \sim\left\langle\chi_{x} \chi_{y}\right\rangle$ which are known as the normal and anomalous 


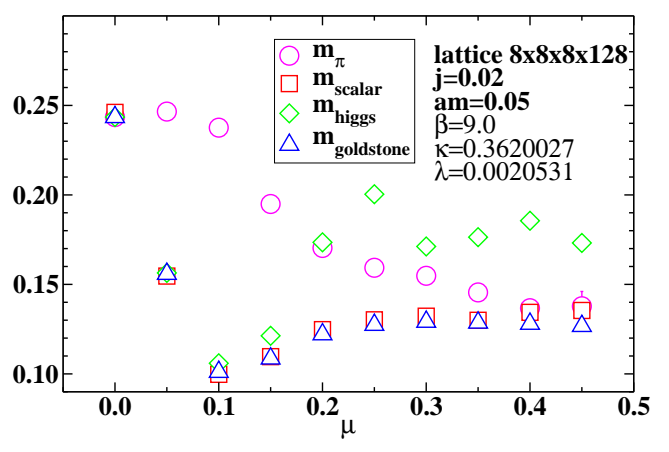

Figure 2: Mass spectrum of various bosonic excitations as a function of $\mu a_{t}$.

parts respectively. On a finite volume $A \equiv 0$ for $j=0 ; \lim _{j \rightarrow 0} \lim _{V \rightarrow \infty} A \neq 0$ signals particle-hole mixing resulting from the breakdown of $\mathrm{U}(1)_{B}$ symmetry, and hence superfluidity. Due to $\mathrm{SU}(2)$ symmetries the only independent components of $\mathscr{G}$ are $\operatorname{Re} N_{11} \equiv N$ and $\operatorname{Im} A_{12} \equiv A$ and their barred counterparts.

We have studied the model with a chemical potential $\mu a_{t}=0.25$, ie. above the critical $\mu_{c}$ required to enter the superfluid phase. All four channels yield clear signals for single particle bound states.

Like any meson constructed from staggered fermions, the correlators in principle describe two states and must be fitted using the form

$$
C(t)=A\left[e^{-m t}+e^{-m\left(L_{t}-t\right)}\right]+B\left[e^{-M t}+(-1)^{t} e^{-M\left(L_{t}-t\right)}\right],
$$

where $m$ and $M$ denote the masses of states with opposite parities. In most cases we find $M \gg m$; however for $\mu>\mu_{c}$ the pion correlator has a distinct "saw-tooth" shape, and in fact the fit yields $m_{\pi}>M_{b 1}$, where $\pi$ denotes the usual pseudoscalar pion, and $b 1$ a state of opposite parity, which must therefore be scalar. In Fig. 目 the corresponding spectrum for all the boson states. Note that all states are approximately degenerate at $\mu=0$; the equality of pion, higgs and goldstone correlators is guaranteed by $\mathrm{SU}(2)$ symmetry at $\mu=0$, but the degeneracy of the scalar in the chirally-broken vacuum can only arise as a result of meson-diquark mixing due to $j \neq 0$. Next, note that the pion mass remains constant for $\mu<\mu_{c}$, where it is a pseudo-Goldstone boson associated with chiral symmetry breaking, and then falls once the superfluid phase is entered; this is in accordance with the predictions of $\chi \mathrm{PT}$. Most of the other states show a much steeper decrease with $\mu$ for $\mu<\mu_{c}$, followed by a gentle rise to a plateau at $m a \approx 0.13$ in the superfluid phase $\mu>\mu_{c}$, precisely as expected of the goldstone state in the superfluid phase with diquark source $j \neq 0$.

The exception is the higgs, which rises more steeply to become the heaviest state at large $\mu$. We conclude that the breaking of degeneracy between higgs and goldstone states is clear supplementary evidence for the breaking of $\mathrm{U}(1)_{B}$ symmetry in the superfluid phase and all states with $J^{P}=0^{+}$ including the $b 1$ but except the higgs have some projection onto the Goldstone state, regardless of whether the original interpolating operator is mesonic or baryonic.

\subsection{Fermionic Spectrum}

We have also studied the fermion spectrum, often in the context of condensed matter called 


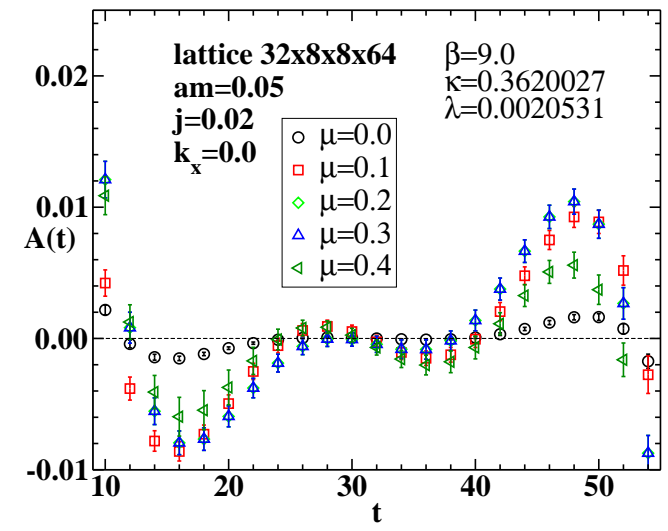

Figure 3: Close-up of $A(t)$ for various $\mu a_{t}$.

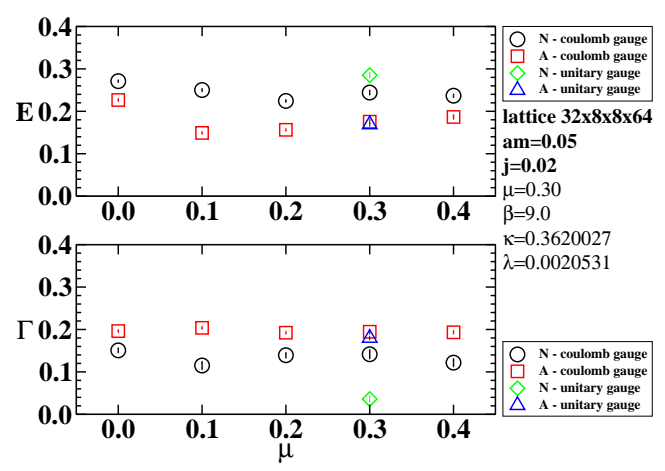

Figure 4: $E$ and $\Gamma$ vs. $\mu a_{t}$ in both normal and anomalous channels.

the quasiparticle spectrum. Since the Gor'kov propagator $\mathscr{G}$ is not gauge invariant we have to specify a gauge fixing procedure. A feature of the quenched approach is that it permits large statistics to be accumulated with relatively little CPU effort. This has enabled us for the first time in a gauge theory context to study $\mathscr{G}$ at $\mu \neq 0$, by helping to overcome the sampling problems associated with gauge fixing. We have experimented with two gauge choices: Unitary gauge $\varphi \mapsto \varphi^{\prime}=\left(0,0, \varphi_{3}^{\prime}\right)$, which is implemented before the reconstruction of the 4th dimension, and is unique up to a $\mathrm{Z}_{2}$ factor, specified by demanding $\varphi_{3}^{\prime} \geq 0$; and Coulomb gauge, implemented by maximising $\sum_{x i} \operatorname{tr}\left(U_{x, i}+U_{x-\hat{\imath}, i}^{\dagger}\right)$, in an attempt to make the gauge fields as smooth as possible and hence improve the signal-to-noise ratio. We have studied the normal and anomalous fermion timeslice propagators on a $32 \times 8^{2} \times 64$ lattice at $\beta=9.0, \kappa=0.1, \lambda=0.0020531, m a=0.05$, $j a=0.0 .2$ and $\mu a_{t}=0.3$; the last value chosen to ensure $\mu>\mu_{c}$. Two features to note are that the Coulomb data is roughly twice as large as the unitary data reflecting an enhanced signal and that there is little variation with $k_{x}$.

In the NJL model the quasiparticle propagator can be successfully fitted using the forms

$$
\begin{aligned}
& N(t)=P e^{-E_{N} t}+Q e^{-E_{N}\left(L_{t}-t\right)}, \\
& A(t)=R\left[e^{-E_{A} t}-e^{-E_{A}\left(L_{t}-t\right)}\right],
\end{aligned}
$$

where for $\mu \neq 0$ there is no reason to expect $P=Q$, but for a well-defined quasiparticle state the equality $E_{N}=E_{A}$ should hold. Here, by contrast, only the anomalous channel fits produced an acceptable $\chi^{2}$ and shows any evidence of gauge independence. The value of $E_{A}$ obtained is very close to $m_{\pi} / 2$, indicating that at this value of $\kappa$ the pion is a weakly bound state. Another striking feature of the data is the approximate forwards-backwards symmetry of $N(t)$, implying $P \simeq Q$. We have studied the dispersion relations $E_{A}\left(k_{x}\right)$ for data taken on a $32 \times 8^{2} \times 64$ lattice; it confirms that the quasiparticle excitation energies are $k$-independent: one motivation for our study was to investigate to what extent the concept of a Fermi surface, which is not strictly gauge invariant, can be put on a firm empirical footing in a gauge theory. Our results shows no evidence for a Fermi surface. The nature of the quasiparticle excitation is clarified a little at the other parameter set studied, namely $\kappa=0.3620027$. In this case our results show no evidence for any well-defined spin$\frac{1}{2}$ state in either normal or anomalous channels; as a result of confinement the excitation spectrum 
of the model seems to be saturated by the tightly bound spin- 0 states of Fig. 2. Fig. 3 shows a closeup of $A(t)$ for various $\mu$ values, showing the presence of an oscillatory component whose amplitude initially grows with $\mu$ (the $\mu a_{t}=0.3$ points overlay those from $\mu a_{t}=0.2$ ), but whose wavelength is roughly $\mu$-independent. The origin of the oscillation could possibly be associated with the nonunitarity of the model, but is most likely a manifestation of independent spin- $\frac{1}{2}$ excitations being ill-defined due to confinement.

We have fitted the $\kappa=0.3620027$ data to the forms

$$
\begin{gathered}
N(t)=P e^{-E_{N} t} \cos \left(\Gamma_{N} t+\phi\right)+Q e^{-E_{N}\left(L_{t}-t\right)} \cos \left(\Gamma_{N}\left(L_{t}-t\right)+\phi\right), \\
A(t)=R\left[e^{-E_{A} t} \cos \left(\Gamma_{A} t+\phi\right)-e^{-E_{A}\left(L_{t}-t\right)} \cos \left(\Gamma_{A}\left(L_{t}-t\right)+\phi\right)\right],
\end{gathered}
$$

where we interpret $E$ as the energy and $\Gamma$ as the width of a quasiparticle excitation. The results' most striking feature is their independence of $\mu$, with $\Gamma$ of the same order of magnitude as $E$. An interesting systematic effect is that $E_{N}>E_{A}$ while $\Gamma_{N}<\Gamma_{A}$, which has motivated us to study $\sqrt{E^{2}+\Gamma^{2}}$ vs. $\mu$ : the disparity between normal and anomalous channels is significantly reduced. Inspection of the $\mu a_{t}=0.3$ data also shows that the gauge dependence of this result is $\mathrm{O}(20 \%)$ at worst. Numerically, $\sqrt{E^{2}+\Gamma^{2}}>m_{\pi}$, indicating strong quark - anti-quark binding, due to the persistence of confinement at this value of $\kappa$. Therefore we can interpret the effect of confinement as rotating the quasiparticle pole into the complex plane, the rotation angle being larger in the anomalous channel than in the normal one.

\section{Conclusions}

Our attempt to alter the nature of the gluon background by changing the parameters of the $3 d$ DR gauge-Higgs model has been a partial success, in that in going from $\kappa=0.3620027$ to $\kappa=0.1$ the strength of the binding between quarks weakens significantly.

However, in neither case is there evidence for significant departure of $n_{q},\langle\bar{q} q\rangle$ and $\langle q q\rangle$ from the behaviour predicted by $\chi \mathrm{PT}$, so that even if quarks are important degrees of freedom at $\kappa=0.1$, there is no evidence for the formation of a degenerate system signalled by SB scaling.

Sadly though, it appears to remain the case that despite its "unreasonable effectiveness" in virtually all other aspects of lattice QCD, the quenched approximation has nothing useful to tell us about the physics of high quark density.

\section{References}

[1] P. Giudice and S. Hands, hep-lat/0703001. Manuscript accepted for publication in Nucl.Phys.B.

[2] M.A. Stephanov, Phys. Rev. Lett. 76 (1996) 4472.

[3] A. Hart and O. Philipsen, Nucl. Phys. B572 (2000) 243.

[4] J.B. Kogut, M.A. Stephanov, D. Toublan, J.J.M. Verbaarschot and A. Zhitnitsky, Nucl. Phys. B582 (2000) 477.

[5] S.J. Hands, S. Kim and J.I. Skullerud, Eur. Phys. J. C48 (2006) 193.

[6] B. Alles, M D'Elia and M.P. Lombardo, Nucl. Phys. B752 (2006) 124. 